\section{Science Lights Up 1991 MRS Spring Meeting}

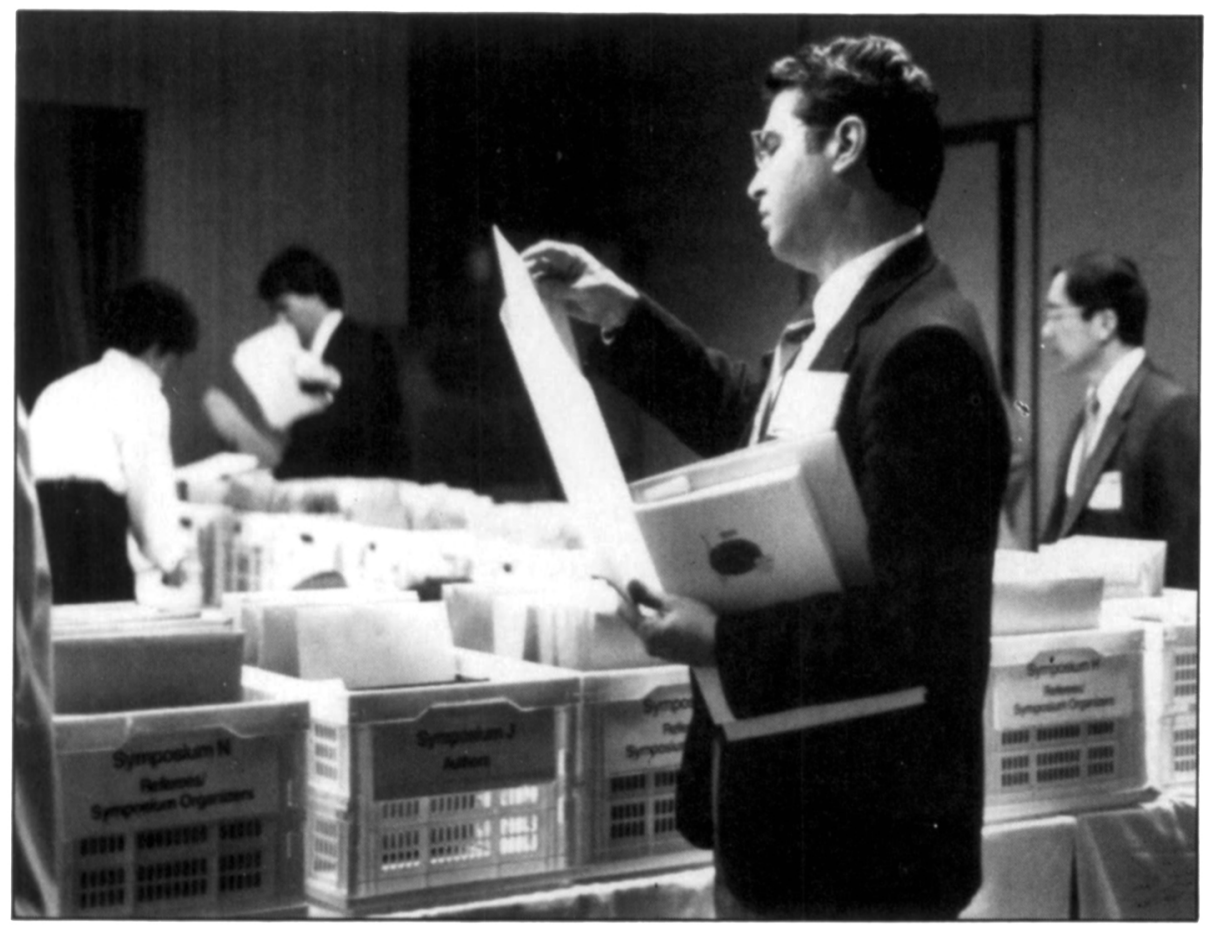

Authors and reviewers check papers in the manuscript room at the 1991 MRS Spring Meeting. Presentations that will be published as part of the MRS Symposium Proceedings series are peerreviewed at the meeting.

The 1991 MRS Spring Meeting in Anaheim beamed with innovative science as well as California sunshine when 1,800 participants convened from April 29 through May 3 for 23 technical sessions, short courses, and an extensive equipment exhibit. The meeting, chaired by A.K. Hays, Sandia National Laboratories, Ernesto E. Marinero, IBM Almaden Research Center, and Carl V. Thompson, Massachusetts Institute of Technology, shed light on a number of new topics and expanded on familiar ones.

- Late News. Light emerging from electrochemically etched porous silicon brought excitement in the late-news session, Visible Light from Porous $\mathrm{Si}$ : An Open Door to Silicon-Based Optoelectronics? As the 400 some attendees filed in, many carried their own chairs to grab a spot in the rapidly filling room. (See sidebar in this report.)

- Plenary Address on the Environment. Plenary speaker Stephen A. Lingle, dep- uty director of the Office of Environmental Engineering and Technology Demonstration, U.S. Environmental Protection Agency, focused on the trend toward developing "environmentally friendly" materials. The EPA's new approach emphasizes pollution prevention in addialso focuses on setting priorities using risk assessment, voluntary compliance and economic incentives, and more reliance on science. (Lingle's address is published elsewhere in this issue.) two special sessions: a lunchtime panel discussion on graduate materials science education and an informal networking session on K-12 science education.

The graduate education panel reached no definitive conclusions as to where materials science education should go, but they did discuss the growing role of scientists and engineers with master's degrees and tion to the usual end-of-pipe treatment. It

- Education. Education was addressed in

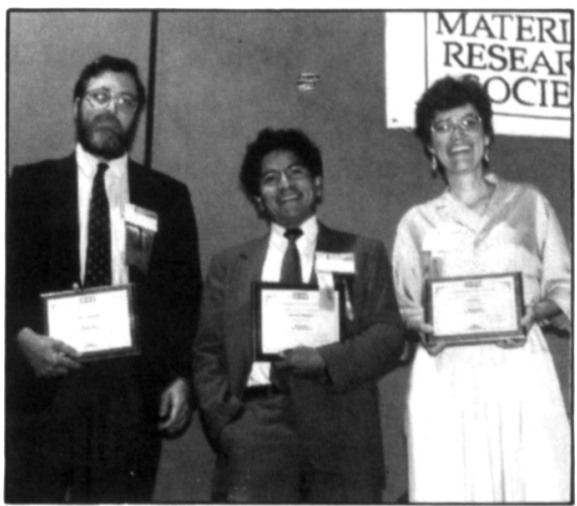

The 1991 MRS Spring Meeting Chairs hold plaques acknowledging their efforts in putting together an interdisciplinary program spanning the breadth of current materials research. Left to right: Carl V. Thompson, Churchill College, Cambridge; Emesto E. Marinero, IBM Almaden Research Center; and A.K. Hays, Sandia National Laboratories.

the need to decrease the pressure on PhD graduates to pursue solely research positions, thereby opening the door for talented people to contribute, for example, to manufacturing needs.

The networking session on K-12 science 


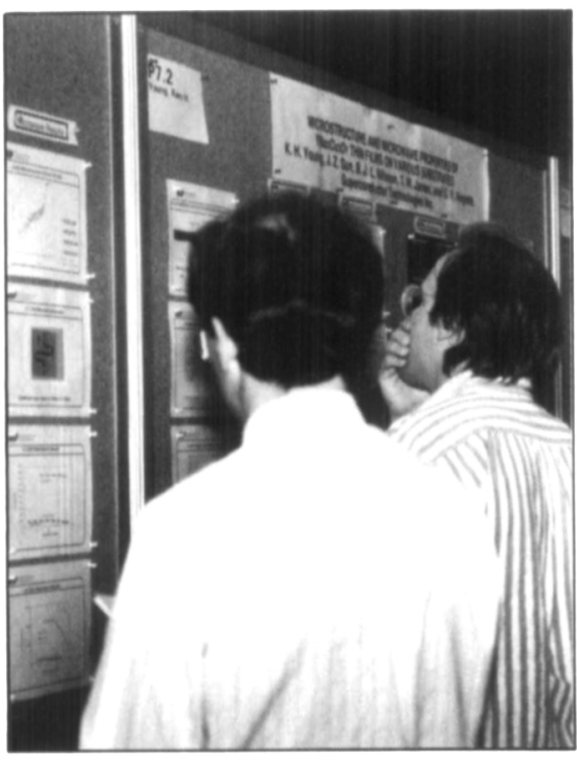

Poster sessions give participants a close-up view of current research. education focused on the role that scien tists and MRS can play to improve science education in the early years. Part of the discussion revolved around whether changes should come from the top down (through legislation, curriculum changes, teacher certification) or from the bottom up (through contact with local schools and teachers). For now, the group favors grassroots efforts as both more manageable and less imposing.

- Technical Sessions. Polymers for applications ranging from electronics and optical storage to sutures, interfaces between every imaginable materials combination, silicon for solar cells and electronics, processing methods, electronics reliability, magnetic and optical materials-all were thoroughly discussed in groups of technical symposia. Also covered in individual symposia were environmentally conscious processing, molecular sieves, tribology, and thermoelectrics.

The research speaks for itself in the following reports, which highlight the technical sessions. More detailed information is available in the published proceeding (listed elsewhere in this issue) or from the symposium organizers. The symposium organizers and invited speakers are listed in the Spring Meeting Preview published in the March MRS Bulletin, p. 54-59.

\section{a-Si:H Performance and} Applications Grow

(See MRS Symposium Proceedings Vol. 219)

Symposium A showed how the amor- phous silicon (a-Si:H) field continues to attract considerable attention for diverse applications such as inexpensive solar cells, active matrix liquid crystal (LC) displays, sensor arrays for facsimile machines, radiation detectors, and high definition television.

Although a-Si:H solar cells hold a premier position in the consumer products area, competition is growing from other technologies in the power application market where efficiency of conversion, stability, and cost are major driving factors. a-Si: $\mathrm{H}$ has shown some instability, which lowers the solar cell performance in the initial 1,000 hours of performance, after which they stabilize. Studying this insta-

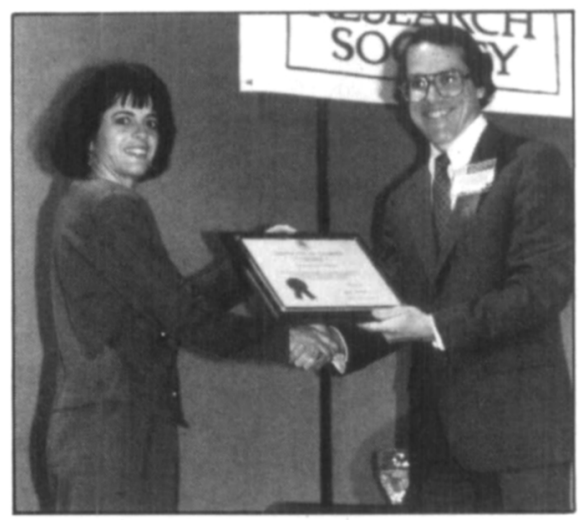

bility from a materials and device point of view has not led to a clear consensus as to its cause, but new modes of deposition (e.g., chemical annealing, Xe-dilution, or hot wire technique) have shown that the photostability effects can be manipulated and reduced.

A review by $\mathrm{G}$. Moddell on a-Si:H in LC spatial light modulators showed that by using a ferroelectric $L C$, a high resolution image of 100 line pairs $/ \mathrm{mm}$ is achievable. This may lead to commercial products ranging from optical computing to high definition television.

Another application of integrated a-Si: $\mathrm{H}$ was reported by the Xerox group. An a$\mathrm{Si}: \mathrm{H}$ photosensing element was configured with four a-Si:H thin film transistors, providing gain at the pixel level. Existing commercial sensing arrays have no gain at the pixel level. The novel design, which requires two global connections and power from scan lines, could be an advantage for $\mathrm{a}-\mathrm{Si}: \mathrm{H}$ in the sensor marketplace.

For large areas, many applications of a$\mathrm{Si}: \mathrm{H}$ require plasma-enhanced chemical vapor deposition using silane gas. J. Schmitt reviewed the design of these systems, focusing on an interrupted plasma, one of the major sources of pin holes in a$\mathrm{Si}: \mathrm{H}$ semiconducting film. This approach could enhance the yield of the products produced.

Prof. Lourdes Salamanca-Riba (left), faculty adviser for the MRS University Chapter at the University of Maryland, receives the newly formed chapter's charter from MRS President James B. Roberto. In 1984, Salamanca-Riba, then a student at the Massachusetts Institute of Technology, received an MRS Graduate Student Award.

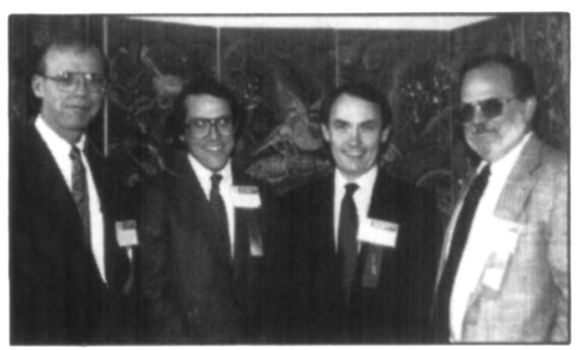

Templates and Surface Relief Aid Heteroepitaxy of Dissimilar Materials

(See MRS Symposium Proceedings Vol. 221)

Heteroepitaxy of dissimilar materials (Symposium C) poses the unique scientific challenge of growing one material on another when the overlayer has a different crystal structure, atomic spacing, type of atomic bonding, etc. As the technological need for a broader range of material combinations grows for applications such as magnetic recording materials, high $T_{c}$ superconductors, and novel metal/ semiconductor heterojunction devices, the old tenet that epitaxy works best only for two very similar materials is giving way to an extensive set of experimental studies aimed at determining how to make heteroepitaxy successful under conditions of extreme dissimilarity.

One solution is to form a substrate tem-

Plenany Speaker Stephen A. Lingle (second from right) from the U.S. Environmental Protection Agency poses with MRS officers (left to right): Slade Cargill, first vice president; James B. Roberto, president; and Russell R. Chianelli, immediate past president. (Lingle's address is published elsewhere in this issue.) plate which will assist in the overgrowth of the upper dissimilar material layer. The conditions to deposit a proper template layer often differ markedly from those used to grow the remainder of the overlayer. The division of the problem into separate template formation and overgrowth stages has proven successful experimen- 
tally in a wide range of heteroepitaxial combinations. In some cases, the template may even consist of a third, carefully chosen intermediate material capable of both growing well on the underlying substrate and properly seeding the overlayer.

One session highlighted how the growth mode of the overlayers can be modified in the presence of intentionally introduced surface adsorbates. For example, the adsorption of arsenic on a silicon surface can change the growth mode of an overlying germanium epitaxial layer from the undesirable islandlike mode to that of layer-bylayer growth.

Different atomic spacing between the substrate and overlayer usually results in a number of undesirable strain effects such as misfit dislocations. One report about GaAs-on-Si showed a dislocation density in the $10^{5} / \mathrm{cm}^{2}$ range, one to two orders of magnitude lower than the best previously reported values. This was achieved by growing the top layer on submicron corrugated patterned substrates with selective oxide coverage attained by the difference in oxide thermal desorption characteristics on different crystallographic planes of the patterned Si substrate.

Theoretical issues included interface formation, initial stages of growth, effect of surface reconstructions, interface charge considerations, and effects of strain and critical thickness. The ability to use epitaxial stabilization to form phases not even stable in bulk were discussed, including several experimental demonstrations such as fcc iron and a ferromagnetic form of $\mathrm{MnAl}$. There was even a discussion of graphoepitaxy, or artificial epitaxy, where single-crystalline films can be deposited on noncrystalline substrates by means of carefully chosen surface-relief patterns reflecting the crystal symmetry of the desired crystalline overlayer.

A distinct trend could be seen in the increasing use of in-situ characterization techniques such as reflection high-energy electron diffraction, low-energy electron diffraction, and x-ray photoemission spectroscopy (and its relatively recent cousin $x$ ray photoemission diffraction). These complement the more traditional ex-situ techniques such as high resolution transmission electron microscopy, photoluminescence, Rutherford Backscattering and $x$-ray diffraction, and are allowing researchers in the field to get a "real-time" view of the heteroepitaxy process.

\section{Atomic Layer Processing Balances Deposition and Etching}

(See MRS Symposium Proceedings Vol. 222)

The symposium on Atomic Layer Growth and Processing (Symposium D) covered fundamental issues and technological applications of atomically controlled processing. Reported advances concern the relationship of surface chemistry and structure to atomically controlled epitaxy of GaAs. Earlier views of the atomic layer epitaxy process, based on surface saturation, have given way to a dynamic model whereby self-limiting growth is a balance between deposition and etching reactions.

Due to their planar structure, high $\mathrm{T}_{\mathrm{c}}$ superconductors appear to be well suited to atomic layer growth techniques. In-situ monitoring techniques both offer insight into the growth process and serve as potential feedback controls to monitor growth progress. Atomic-layer-growth-based materials have both electronic device and solar cell applications. Atomically controlled processes have also been extended to the controlled oxidation and etching of semiconductor materials, offering potential for highly uniform processes controllable down to the atomic $(\mathrm{nm})$ level.

\section{Superconductivity Research Focuses on Grain Boundaries}

Symposium P, Interfaces in High Temperature Superconducting Systems placed special attention on the structure and properties of grain boundaries and the earliest stages of growth in thin films.

The mechanism by which grain boundaries degrade the superconducting properties in high temperature systems remains unresolved. For $\mathrm{YBa}_{2} \mathrm{Cu}_{3} \mathrm{O}_{x^{\prime}}(\mathrm{YBCO})$, it is known that the critical current across a grain boundary decreases rapidly with increasing grain boundary angle. High resolution TEM images of low-angle boundaries in YBCO clearly show a defect structure consisting of alternating regions of distortion or disorder (associated with grain boundary dislocations) and regions of lattice-matched material across the boundary. Assuming that the wellmatched regions represent the conduction path across the boundary, the total area for conduction decreases rapidly with increasing grain boundary angle, approaching zero around $10^{\circ}$. This suggests that for high-angle boundaries, the conduction is through the disordered material, presumably by Josephson coupling. Other work did show that some special high-angle boundaries provide high current conduction across the boundary.

High-angle boundaries are being engineered for use as Josephson junctions in electronic devices. Very narrow $(0.5 \mu \mathrm{m})$ superconducting quantum interference devices (SQUIDS) have been fabricated using $\mathrm{SrTiO}_{3}$ bi-crystals as a template to create grain boundaries with controlled misorientation angles in YBCO thin films.
SQUIDS made from $24^{\circ}$ grain boundaries showed the lowest white-noise level (about 450 times Plank's constant).

A novel method to introduce a $45^{\circ}$ grain boundary in YBCO thin films was also presented. It was found that YBCO grown on a $\mathrm{SrTIO}_{3} / \mathrm{MgO}$ bilayer on sapphire is rotated $45^{\circ}$ with respect to YBCO grown on $\mathrm{SrTiO}_{3}$ on sapphire. This process makes it possible to produce grain boundary Josephson devices without using bi-crystals, and to locate the junctions by patterning the initial MgO layer.

Work was also presented on the mechanisms by which thin films initially grow on both lattice-matched and lattice-mismatched substrates. Both STM and TEM clearly show that on a lattice-mismatched substrate like $\mathrm{MgO}, \mathrm{YBCO}$ nucleates as individual islands and then grows to coalescence. STM shows that the growth mechanism is by screw dislocations with one-unit-cell-high ledges. For latticematched substrates like $\mathrm{SrTiO}_{3}$, the mechanism is not as clear, but a more layer-by-layer growth may occur. The resulting film morphology is strongly affected by growth rate, which can determine the density of misoriented islands that nucleate.

\section{Rapid Thermal Processing Used for Engineered Dielectrics \\ (See MRS Symposium Proceedings Vol. 224)}

The greatest interest in rapid thermal and integrated processing (Symposium $\mathrm{F}$ ) seems to be in using RTP for the growth of dielectrics for gates and capacitors. "Engineered dielectrics" will be critical to meet upcoming requirements for high density circuits. Stacked nitrided oxides or reoxidized nitrided oxides (ONO) offer much better performance than simple $\mathrm{SiO}_{2}$ dielectric films. RTP is an ideal way to fabricate these structures, and there were several discussions of novel techniques using $\mathrm{N}_{2} \mathrm{O}$ and $\mathrm{NF}_{3}$ for annealing, nitriding, and pre-cleaning. Work in this area is proceeding very quickly and it bears watching closely in future meetings.

Temperature measurement is always a concern for RTP. Some encouraging new developments indicate that soon one might really be able to determine the temperature of a wafer during RTP. Work on this subject now appears to be focused and taken seriously.

Among the remaining topics, rapid thermal CVD to deposit films generated the most papers and interest, particularly for depositing silicon and Si-Ge alloys. Although these processes are not worthy of large-scale manufacturing now, they hold great promise for the integration of several 
process steps to more tightly control interfaces between layers.

\section{Photorefractive Polymer, Novel Optical Storage Methods Brought to Light \\ (See MRS Symposium Proceedings Vol. 228)}

Significant advances in nonlinear optical devices and conceptually exciting optical storage methods were among the highlights of Symposium M, Polymeric Materials for Integrated Optics and Information Storage. Part of the symposium was held jointly with Symposium N, Materials for Epitaxial Information Processing.

The photorefractive effect has been observed in a polymeric material for the first time, as reported by W.E. Moerner (IBM Almaden Research Center). Moerner and co-workers synthesized an epoxy-based polymer containing nonlinear chromophores and hole transport agents. The material exhibited the ability of erasable holographic storage, and its diffraction efficiency can be tuned by the external electric field.

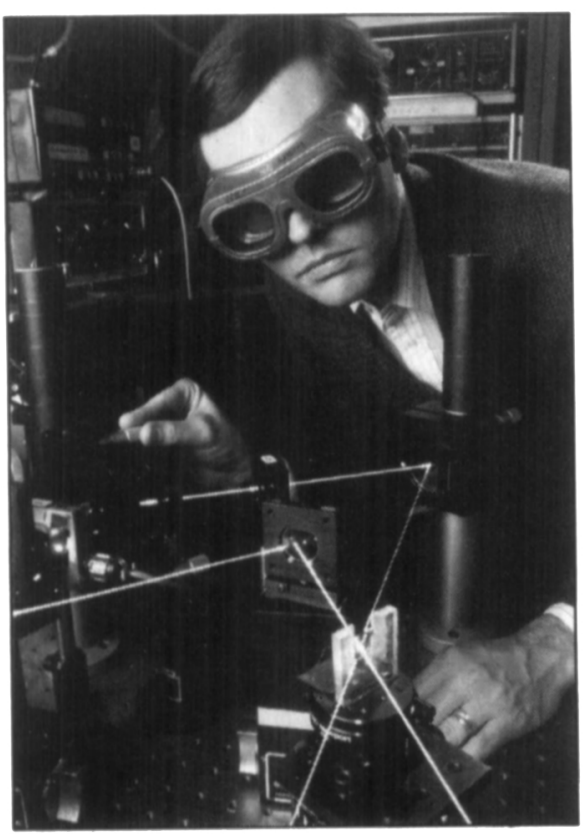

A demonstration of the photorefractive effect observed for the first time in a polymeric material. W.E. Moerner and co-workers at IBM's Almaden Research Center synthesized an epoxy-based polymer containing nonlinear chromophores and hole transport agents. The material exhibited the ability of erasable holographic storage, and its diffraction efficiency can be tuned by the external electric field.
Significant progress in device performance was reported by G.F. Lipscomb (Lockheed Photonics and Lightwave Technology group). The Lockheed workers have fabricated a Mach-Zehnder modulator that can switch light on and off at frequencies up to an impressive $20 \mathrm{GHz}$.

Unusual methods of optical storage sparked interest in the symposium. Two television cameras were rolling as $\mathrm{P}$. Rentzepis (University of California, Irvine) described a three-dimensional photochemical storage method first proposed in Science (August 25, 1989). A photochromic compound is dissolved in a polymer cube. Storage, accomplished by a two-photon process, occurs only where two laser beams intersect. The data are read by fluorescence. Rentzepis' photos of cubic media with colored dots written in them generated significant press interest, given the potentially enormous storage densities that any three-dimensional method can claim.

A. Fujishima of the University of Tokyo's Department of Synthetic Chemistry described another unusual storage method. Reversible chemistry can be achieved in azobenzene derivatives by light-induced trans-cis isomerization followed by electrochemical reduction to a hydrazobenzene, which can be electrochemically oxidized back to the starting trans isomer. Fujishima pointed out that the electrochemical steps can be carried out with a scanning tunneling microscope tip as the electrode, allowing huge storage densities.

Several other fascinating photochromic materials were described in the optical storage session, including diarylethenes and fulgides with high cyclability, by $M$. Irie (Kyushu University) and R. Matsushima (Shizuoka University), respectively. G. Ashwell (Cranfield Institute of Technology, U.K.) reported an unusual zwitterionic Langmuir-Blodgett film material that exhibits both photochromic and nonlinear optical behavior. These properties increase with the number of monolayers, and films of arbitrary thickness can apparently be prepared given enough time.

R.J. Twieg (IBM) introduced the nonlinear optical area, describing chromophores and polymers that can be used to fabricate waveguide devices. Twieg's colleagues B.A. Smith and J.D. Swalen, described successive steps in the process that goes from chromophore synthesis to device performance. R.N. DeMartino (Hoechest Celanese Corporation) reported the synthesis of a polymer containing dimethylaminonitrostilbene as the nonlinear chromophore. DeMartino and co-workers have measured a second-order susceptibil- ity of $165 \mathrm{pm} / \mathrm{V}$ for the material, and have fabricated a modulator. G. Stegeman (University of Central Florida) gave an overview of the device area, listing as the current limiting factors waveguide fabrication and the ability to predict third-order susceptibilities. He appealed to the theoretical community for greater involvement in this difficult area.

In the area of III-V and II-VI materials and devices for optical applications (Symposium $N$ ), the emphasis was on molecular beam epitaxial (MBE) growth and device fabrication. J.P. Harbison (Bellcore) reported on vertical cavity surface emitting lasers suitable for optoelectronic integration and 2-D signal processing, and L.M.F. Chirovsky (AT\&T Bell Laboratories) described the use of symmetric selfelectrooptic effect devices made of GaAs/AlGaAs multiple quantum wells for optical information processing. C.S. Hong (Boeing Electronics) reported the use of low threshold quantum-well lasers in optoelectronic backplane applications, and M.C. Wu of Bell Laboratories discussed the use of ultrashort optical pulses for optical communication applications. Repetition rates as high as $350 \mathrm{GHz}$ have been demonstrated using InP/InGaAsP. M.T. Asom (AT\&T Bell) described the molecular beam epitaxial growth requirements for infrared photodetector devices. Control on the order of $1 \mathrm{~mm}$ is required. R.N. Bhargava (Phillips Laboratories) described recent progress in blue lasers for optical memory applications. Frequency doubling electron-beam-pumped $\mathrm{ZnSe}$ and pn junction lasers were all analyzed in detail. It was clear that blue lasers from frequency doubling have immediate applications. Many papers on p-type doping of $\mathrm{ZnSe}$ were also presented.

\section{Polymer Degradability Tailored for Diverse Applications}

Most plastics and fibers are organic materials, and like all organic materials, have a finite useful lifetime. The ability to predict and control the lifetime allows optimizing material properties to fit a particular application. Polymer Lifetimes, Symposium L, addressed the use of polymeric materials over a wide range of applications with lifetimes from a few days (bioresorbable polymers, B.L. Benicewicz, Los Alamos National Laboratory) to the 30-year lifetime of the space station (LED environment, S.L. Koontz, NASA Johnson Space Center).

The theme of the symposium was the multidisciplinary nature of lifetime predicting and controlling polymer lifetimes. This involved such diverse topics as the chemical pathways to polymer degradation (e.g., 


\section{Graduate Student Award Finalists 1991 MRS Spring Meeting}

Yih Chang, Stanford University, Paper R1.11, "Direct Evidence of the Si Interstitialicy Injection in Si During Pulsed Laser Melt Process"

*David K. Fork, Stanford University, Paper P4.2, "Epitaxial YBCO and Other Oxides on Si and Si-onSapphire by Pulsed Laser Deposition"

*Imran Hashim, California Institute of Technology, Paper T3.6, "Magnetic and Structural Properties of Tantalum-Permalloy Multilayer Films"

Jian Li, Cornell University, Paper R5.5, "Kinetics of Nucleation and Growth, Metastable Phase Formation and Oxygen Diffusion Induced Grain Boundary Migration in $\mathrm{Cu}$ Oxide Thin Films"

Yi-Qun Li, Stevens Institute of Technology, Paper P7.26, "Effect of Composition on Surface Morphology, Microstructure and Superconductivity of $\mathrm{YBa}_{2} \mathrm{Cu}_{3} \mathrm{O}_{7 \times}$ Thin Films Deposited by Metalorganic Chemical Vapor Deposition"

*Yun-Fook Liew, Rensselaer Polytechnic Institute, Paper S14.12, "Morphology and Magnetic Phase Transitions of Monolayer-Range $\mathrm{Fe}$ Films on $\mathrm{Au}(001)^{\prime \prime}$

Hsueh Yi Lu, Northwestern University, Paper E3.3, "The Role of Dilution Gas in Plasma-Deposited Amorphous Hydrogenated Carbon"

Wei-Xin Ni, Linkoping University, Paper B3.5, "Model Calculations of Dopant Surface Segregation During Growth of Silicon Films by Molecular Beam Epitaxy"

*Wei Xia, University of California, San Diego, Paper N4.2, "Compositional Disordering of Superlattices by Solid-Phase Regrowth"

\section{* Graduate Student Award Recipient.}

defect structures in PVC; W.H. Starnes, College of William and Mary) and the methodology for monitoring electrical aging (mica-epoxy machine insulation, J.K. Nelson, Rensselaer Polytechnique Institute).

The first session dealt with the mechanistic principles of lifetime prediction, from both chemical and engineering viewpoints. For example, P. Klemchuk (CibaGeigy) described the options available to the materials scientist to either increase or decrease the lifetime of polymers through additives. The themes developed in this first session recurred frequently in the following sessions, which dealt with more specific applications.

The second and third sessions covered the broad topic of materials for electrical applications, clearly demonstrating the ability of polymers to perform both as both conductors (polyanilines, A.G. MacDiarmid, University of Pennsylvania) and insulators (polyethylene, R.J. Densley, National Research Council of Canada).

The final session stressed two very different environments, space and marine, but again considerable common ground emerged regarding the principles required to optimize materials. Conventional wisdom was shown to be an unreliable predictor of polymer lifetime in seawater, but once the mechanism of degradation was understood, controlled lifetime polymers for applications such as fishing nets could be developed (marine applications, K.E. Gonsalvez, University of Connecticut). The spacecraft environment also provided some unpleasant surprises for materials scientists, but again an understanding of mechanisms has allowed the development of highly effective and long-lived polymeric materials (e.g., lifetime prediction, Koontz and mechanistic concerns, J.B. Cross, Los Alamos National Laboratory).

\section{Ion Beam and Plasma Sources}

Modify Superconductors, Polymers, Metals, and Semiconductors

(See MRS Symposium Proceedings Vol. 223)

Symposium E, Low Energy Ion Beam and Plasma Modification of Materials showed the wide range of materials which can be modified advantageously by low energy particles from ion beam and plasma sources.

Fundamental ion-material interactions were highlighted by two videotapes of computer simulations, one of epitaxial $\mathrm{Si}$ growth from Si ions of energy $10-20 \mathrm{eV}(\mathrm{M}$. Kitabatake, Matsushita Electric Industrial Company, Ltd.) and one of carbon ion deposition at 10-50 eV (B. Pailthorpe, University of Sidney). The simulations showed a $10 \mathrm{eV}$ ion setting the target surface into oscillation, then finding an epitaxial position. J. Biersack (Hahn-Meitner Institute) presented an overview of Monte Carlo simulations, including his recent extension of the widely used TRIM code to model the growth of thin films under simultaneous ion bombardment.

Recent developments in electron cyclotron resonance (ECR) sources and microwave-excited plasma sources were reviewed by L.A. Berry (Oak Ridge $\mathrm{Na}$ tional Laboratory), clarifying the conditions of resonance that produce high ion densities at low energy, a combination difficult to achieve in other plasma devices. Ion densities up to $10 \%$ of the gas density are achievable at an input of only $100 \mathrm{eV}$ per ion.

In a joint session with Symposium P, Interfaces in High-Temperature Superconductors, J. Kwo (AT\&T) and A. Tsukamoto (Hitachi) demonstrated the application of $\mathrm{ECR}$ sources to the synthesis of $\mathrm{YBaCuO}$ and $\mathrm{HoBaCuO}$. This improved method for oxygen incorporation enables synthesizing these perovskite materials at temperatures as low as $500-680^{\circ} \mathrm{C}$. A.F. Hebard (AT\&T) described how ion beam etching at a glancing angle may be used to thin hightemperature superconductors to as low as a few lattice constants. A session on III-V materials included the application of ECR sources to the epitaxial growth of GaAs at temperatures as low as $350^{\circ} \mathrm{C}$ on GaAs and $400^{\circ} \mathrm{C}$ on Si (N. Kondo, NTT). Selective growth of $\mathrm{GaAs}$ at $630^{\circ} \mathrm{C}$ was also demonstrated.

Ion beam techniques have dramatically improved the quality of optical thin films. Techniques for controlling density and refractive index were reviewed by U.J. Gibson (Dartmouth College) and recent results on modulating the index of $\mathrm{ZrF}$, from 1.5 to 1.8 by oxygen ion bombardment were shown. This approach is suitable for the fabrication of graded index (GRIN) structures while maintaining planarity. Ferroelectric materials share many processing challenges with the family of high-temperature superconductors. A.I. Kingon (North Carolina State) showed how controlled ion beam sputtering allows optimized deposition from dual targets of $\mathrm{KO}_{2}$ and $\mathrm{Nb}$ to produce high quality films of ferroelectric $\mathrm{KNbO}_{3}$ at $500-600^{\circ} \mathrm{C}$.

Polymers are also easily modified by ion bombardment. S.E. Molis (IBM) reviewed the effects of $500 \mathrm{eV}$ argon ion bombardment of polyamide and showed how infrared spectroscopy reveals a shift to a more carbon-rich surface layer which is 1,000 times more resistant to water absorption than nonbombarded polyamide. 
The symposium concluded with a session on metal film modification. A talk on multilayer magnetic films (M. Senda, NTT) showed how magnetic anisotropy and coercivity respond dramatically to changes in ion beam deposition conditions.

\section{Thermoelectric Efficiency Increases, But Not Without Limit}

(See MRS Symposium Proceedings Vol. 234)

Symposium V, Modern Perspectives on Thermoelectrics and Related Materials, attracted substantial international interest, including representatives from the Soviet Union, France, Germany, Japan, Israel, and the United States. The symposium covered a wide range of theoretical and experimental advances in the use of thermoelectrics for power generation, refrigeration, and sensors.

Recent experimental and theoretical results, as well as a review of the historical development of silicon-germanium technology for thermoelectric applications were presented. Recent advances promise lower cost and more efficient silicongermanium thermoelectric power supplies, particularly for space applications.

L. Anatychuk and L. Bulat (Institute of Thermoelectricity, Chernovsty, U.S.S.R.) revealed several innovative developments in thermoelectricity previously unknown outside the Soviet Union. Highly sensitive microcalorimetry sensors have been developed utilizing a novel, junctionless device principally based on the anisotropy within a single thermoelectric material. Other results concerned methods for major improvements in conversion efficiency and the preparation of high quality thermoelectric materials.

A new fundamental theoretical result concerning the efficiency of thermoelectric devices was reported by D.J. Bergman (Tel Aviv University). Using a new variational procedure, Bergman showed for the first time that a composite of two thermoelectric materials will never result in higher efficiency than can be achieved with the best of the two materials alone.

\section{Molecular Sieve Research Turns to Basics}

(See MRS Symposium Proceedings Vol. 233)

The early history of molecular sieve materials research and development largely centered on microporous aluminosilicates (zeolites), but that picture has changed markedly during the past decade, as was clearly evidenced in the papers presented at Symposium U, Synthesis/Characterization and Novel Applications of Molecular Sieve Materials. While past studies of the catalytic activity of molecular sieves emphasized the properties of the acidic sites, now basic sites in molecular sieves and related materials are gaining attention. The relationship between basicity and sieve structure and between basicity and selectivity were presented and discussed together with possible uses of highly basic sieves in consecutive condensation reactions. The application of molecular sieve materials as catalysts for oxidation reactions and in the synthesis of fine chemicals was described in accompanying talks.

The session on synthesis of molecular sieve structures was highlighted by the report of a 20-ring synthetic gallophosphate named "cloverite" because of the cloverleaf shape of the 20-ring aperture. This structure now surpasses the 18-ring aluminophosphate VPI- 5 synthesized several years ago. Other papers in this session reported on the many emerging approaches for pillaring clays and related layered materials, including new strategies for simultaneously controlling both interlayer spacing and acidity.

Papers in two other sessions addressed the novel application of molecular sieves as hosts for macromolecular entities with unique optical, electron transport, and catalytic properties. Semiconducting metal sulfide clusters, heterobimetallic complexes, and conducting polymers can now be synthesized within the pores and channels of crystalline molecular sieves or intercalated into pillared and layered materials.

Hydrothermal, sol-gel, micro-manipulation, and electrochemical methods for preparing thin film structures with molecular sieving properties were also reported. These novel structures are expected to see wide application in sensor instruments and electronic devices.

Other researchers described recent successes synthesizing nanocrystalline molecular sieve particles, molecular sieves with three-membered rings, and molecular sieves containing major amounts of transition metal ions in the framework. Molecular sieves synthesized in the presence of selected rare earth-macrocyclic ligand complexes have been used successfully as contrast agents for magnetic resonance imaging of the digestive tract. Three talks described the use of zeolites as precursors or components of monolithic ceramics and ceramic membranes; applications include packaging of integrated circuits, structural ceramics, and materials separations.

\section{Complex Mixing Behavior Found in Polymer Blends}

In Symposium K, Polymer Alloys, both
N. Balsara (Exxon) and B. Crist (Northwestern University) reported intriguing experiments concerning the miscibility and phase behavior of blends in which the two components were both randomly branched, but by different amounts, and where either one or none of the components was deuterated. Remarkably complex mixing behavior was found in which even many qualitative aspects were extremely sensitive to subtle variations in branch content and deuteration. Experimental results presented indicate a fundamental nonadditivity of effects with regard to the phase behavior of branched polymer blends. The complete lack of microscopic understanding of these important phenomena was emphasized.

J. Curro (Sandia National Laboratories) described recent theoretical results using a polymer integral equation approach. For isotopic blends, a novel, nonclassical scaling law for the molecular weight dependence of the critical phase separation temperature was discussed. For blends with both unfavorable enthalpic interactions and modest structural asymmetries, the novel scaling law was found to persist, but the standard incompressibility approximation was found to fail dramatically due to a strong coupling of concentration and volume fluctuation processes.

Surprisingly complex behavior was reported in the session on blend dynamics. T. Lodge (University of Minnesota) described scattering measurements on low molecular weight polymer mixtures in which strong, non-mean-field dynamical slowing down effects were found even far removed from the static critical region. P. Green (Sandia) presented data on the diffusion and viscoelastic properties of dense entangled polymer mixtures that exhibited striking nonideal additivity behavior. The potential importance of both strongly modified local segmental dynamics in polymer alloys and dynamical concentration fluctuation effects on entanglement was emphasized, along with our present primitive theoretical understanding of such issues.

In the session on copolymers, G. Fredrickson (University of California, Santa Barbara) presented a new statistical mean field theory of copolymer liquids characterized by chemical microstructural disorder quenched in at the time of polymerization. Extremely rich and exotic phase behavior was predicted to occur as a function of the detailed nature of the copolymer microstructure. A microscopic understanding of such effects could have a major impact on designing new polymer alloys with controlled phase behavior, domain size, and morphologies. 


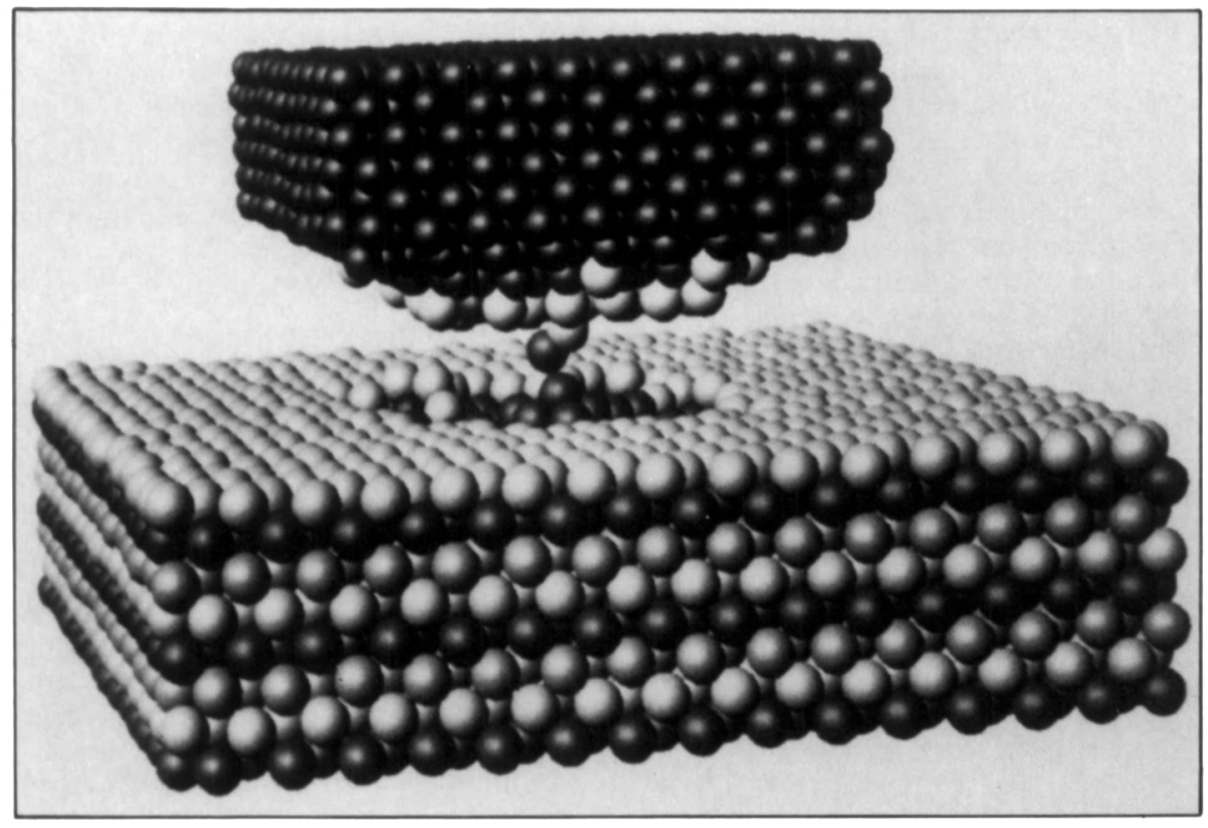

Molecular dynamics computer simulations of the generation of a connective neck developing on separation of a nickel tip (black balls, top) from adhesive contact with a gold surface (bottom slab; topmost gold layer represented by light balls, second layer by dark ones, etc.). Note the adherence (wetting) of a gold layer to the bottom of the nickel tip.

Interest Grows Toward Reliable, Contamination-Free Electronics (See MRS Symposium Proceedings Vols. 225 and 226)

As electronics technology drives toward higher density devices with high performance, electronic and mechanical reliability and contamination reduction become increasingly important. These areas were directly addressed for the first time at an MRS meeting in a group of three symposia, Reliability Issues (G), Mechanical Behavior of Materials and Structures $(\mathrm{H})$, and Materials Contamination Control in Microelectronics (I).

Improvements in process and equipment contamination control $(90 \%$ of the particle contamination problem by 1995) will result in improvements in equipment utilization, said V. Menon of Sematech in the opening presentation on contamination control in microelectronics. Menon presented results that indicate $37 \%$ of particle problems come from wet cleanups; this emphasizes the need for dry cleanup processes. He also believes that moisture in vacuum process systems is the largest single cause of particle formation in such equipment, e.g., plasma metal etch. He encouraged development of in-situ particle detection.

A. Slaoui(CRN, France) showed that minority carrier diffusion length is a good measure of surface metallic contamination. M. Ray (MCNC) gave information on testing of a promising perfluorinated hydrocarbon chemical for use in polysilicon etch residue removal. K. Maex (IMEC, Belgium) presented results of a TXRF study of $\mathrm{Fe}, \mathrm{Cu}, \mathrm{Ni}$, and $\mathrm{Zn}$ on silicon and silicon dioxide surfaces in $\mathrm{NH}_{4} \mathrm{OH} / \mathrm{H}_{2} \mathrm{O}_{2}, 2 \% \mathrm{HF}$, buffered $\mathrm{HF}$, and DI water. On silicon, with a native oxide, $1 \mathrm{ppb}$ Fe in $\mathrm{NH}_{4} \mathrm{OH}$ leaves $1 \times 10^{12} \mathrm{Fe} / \mathrm{cm}^{2}$. No detectable $\mathrm{Fe}$ is added in HF. Trace amounts of Fe are added in DI water. No detectable $\mathrm{Cu}$ addition was observed in $\mathrm{NH}_{4} \mathrm{OH}$. $\mathrm{Cu}$ around $1 \times 10^{12} / \mathrm{cm}^{2}$ was added in DI water, and around $3 \times 10^{11} /$ $\mathrm{cm}^{2}$ in $\mathrm{HF}$ solutions. For $\mathrm{Ni}, 100 \mathrm{ppb}$ in $\mathrm{NH}_{4} \mathrm{OH}$ gave $1 \times 10^{13} / \mathrm{cm}^{2}$, the same as $\mathrm{Fe}$ at $1 \mathrm{ppb}$. For $\mathrm{Zn}, 100 \mathrm{ppb}$ in $\mathrm{NH}_{4} \mathrm{OH}$ gave $5 \times 10^{12} / \mathrm{cm}^{2}$. In $\mathrm{HF}$, no $\mathrm{Ni}$ or $\mathrm{Zn}$ additions were detected.

Particles in plasmas is a growing area of research. Five papers on this topic all centered on the possible mechanism of particle formation and forces acting on particles in process plasmas. One group believes an ion drag force helps move particles to the plasma sheath. Regarding particle sizes, one group found that $60 \%$ of particles in the studied plasma are $<0.5 \mu \mathrm{m}$, and $90 \%$ are $<0.8 \mu \mathrm{m}$. Two separate talks (Tohoku University and MMEC Belgium) focused on correlation of gate oxide breakdown to surface microroughness as measured by an atomic force microprobe. Both groups concluded that microroughness induced by $\mathrm{NH}_{4} \mathrm{OH} / \mathrm{H}_{2} \mathrm{O}_{2} / \mathrm{H}_{2} \mathrm{O}(1: 1: 5)$ cleaning causes a measurable oxide breakdown degradation. One group suggested that using a $0.05: 1: 5$ mixture to solve this decreased $\mathrm{NH}_{4} \mathrm{OH}$ concentration lowers particle removal efficiency. They indicated that 0.25:1:5 mixtures sufficiently improve microroughness while maintaining good particle removal.

Reliability depends not only on contamination but also on understanding and controlling electromigration, stress-induced damage, the mechanical behavior of thin films, electronic packaging, solder materials and interconnects, and choosing appropriate modeling techniques to study and predict behavior.

\section{Computer Simulations Show Adhesion, Friction on an Atomic Scale}

Friction research has come a long way since the Egyptians fought friction while dragging building materials for the pyramids. Now, researchers are looking at the fundamentals of solids in contact all the way down to the atomic level by watching the interaction of individual atoms in computer simulations. Techniques such as atomic force microscopy and scanning tunneling microscopy have opened new pathways to "see" what happens as atom meets atom.

In Symposium O, Molecular Tribology, U. Landman (Georgia Institute of Technology) showed an example of what computer simulations can tell about solids in contact. He showed a computer simulation of a nickel tip coming into contact with a slab of $\mathrm{Au}$. As a nickel tip comes within $2 \AA$ of a gold surface, the gold wets the nickel, leaping into contact with the nickel and diffusing into the nickel. If the tip is indented and then raised up (at room temperature), a wire $40 \AA$ long may form, connecting the two surfaces. The thin neck between the surfaces is crystalline and forms epitaxially on the nickel. At higher temperatures the neck can grow to be 300 or $400 \AA$.

\section{Materials Processing and \\ Environmental Concerns Merge}

Waste assessment, waste minimization, recycling, and responsible waste disposal are some of the issues companies need to address as environmental problems take the spotlight and as local, state, and federal regulations mount. For the first time, an MRS symposium addressed this issue in Symposium W, Environmentally Conscious Materials Processing.

D. Genser (Allied-Signal) outlined how 
environmentally conscious manufacturing $(E C M)$ can be achieved by knowing what materials are used and how they are processed, assessing current waste handling procedures, and generating new options. $\mathrm{He}$ also defined the expected stages of growth toward ECM: unawareness, initiation, awareness, acceptance, and maturity.

In many cases minor adjustments in a procedure or piece of equipment can greatly benefit the environment while also creating major savings for the company. For example, Allied-Signal saved a halfmillion dollars and reduced the amount of chlorofluorocarbons (CFCs) it uses by 150,000 pounds $(53 \%)$ just by cleaning newly manufactured parts in mild aqueous detergent solutions instead of the ozone-depleting CFCs.

IBM has replaced CFCs as the carrier for lubricant deposition in thin-film disk manufacture with Fluorinert FC-72. They found that this gives a wider process window, faster throughput, and improved uniformity. FC-72 is more expensive, but with conservation, they expect to bring the cost down.

The U.S. Department of Energy has identified eight major waste categories from Sandia and Los Alamos National Laboratories and from five manufacturing sites. Each waste stream has an identified manager who prioritizes and manages waste minimization projects.

D. Allen (University of California-Los Angeles) pointed out one of the difficulties of waste minimization-all the implications of a change must be measured. Sometimes minimizing one form of waste may simply increase another form. For example, incineration substantially reduces the volume of solid waste that must be landfilled, and it may be used to generate energy. However, to prevent the exhaust from reaching the environment, scrubbers are used, producing large amounts of waste water that must be handled.

\section{Metals Behave Differently on \\ Reconstructed Gold Surfaces}

(See MRS Symposium Proceedings Vol. 229)

Atoms on the top layer of a metal crystal rearrange themselves in ways that can profoundly affect the metal's surface properties. Scanning tunneling microscope (STM) studies by D.D. Chambliss and coworkers of IBM, Almaden Research Center have revealed new ways this occurs. This work, presented in Symposium Q, Structure/Property Relationships for Metal/Metal Interfaces, contributes to the fundamental understanding of the surface science that underlies commercially important surface processes, such as epitaxial growth of thin metal films.
STM images show that the top layer of the close-packed (111) surface of crystalline gold buckles (or reconstructs) to form a "herringbone" pattern of zig-zagging ridges only $0.15 \AA$ high. The repeat period of this reconstruction $(280 \AA)$ is 100 times larger than the size of individual atoms. The reconstructed gold surface has regularly spaced surface-lattice dislocations at the corners of the herringbone pattern, where the gold atoms do not nest in their favored geometry.

When less than a monolayer of nickel or iron is evaporated onto the reconstructed gold surface, the deposited atoms aggregate first at the corners of the herringbone pattern. As a result, an array of metal islands forms, each with about 200 atoms, 73 $\AA$ apart in one direction and $140 \AA$ apart in the other. This distance lies between lattice spacings in typical crystalline materials (about 1 to $10 \AA$ ) and the smallest size currently possible for commercially important fabricated features, such as microlithographic structures and magnetic domains (about $1,000 \AA$ or 0.1 micron).

Silver atoms deposited similarly behave very differently. Instead of aggregating at sites determined by the reconstruction, the silver atoms gather at the atom-high steps, which are typically $1,000 \AA$ apart on this otherwise flat single crystal surface. The silver regions appear to grow by diffusioncontrolled aggregation to form distinct "fingerlike" shapes. While such patterns have been predicted from mathematical theories and observed for growth on a macroscopic scale, this is the first time such patterns have been seen at near-atomic length scales. Detailed examination of this pattern shows it is somewhat influenced by the herringbone reconstruction: Finger growth occurs more readily along the "grain" of the pattern than across it.

Taken as a whole, these studies demonstrate that metals display an unexpected variety of aggregation behavior. Even on an atomic scale, where one might expect similarities in such simple deposition experiments, a diversity of atomic patterns is evident. Eventually, studies of this type could lead us to discover new ways to control surface structures and their properties on an ultrasmall scale.

\section{Phase Transformations Take Many Forms}

(See MRS Symposium Proceedings Vol. 230)

Fundamental mechanisms in crystal growth and nucleation as well as amorphization took center stage in Symposium R, Phase Transformation Kinetics in Thin Films. The mechanism and characteristics of silicon crystal growth from or through metals exhibiting low eutectic tempera-

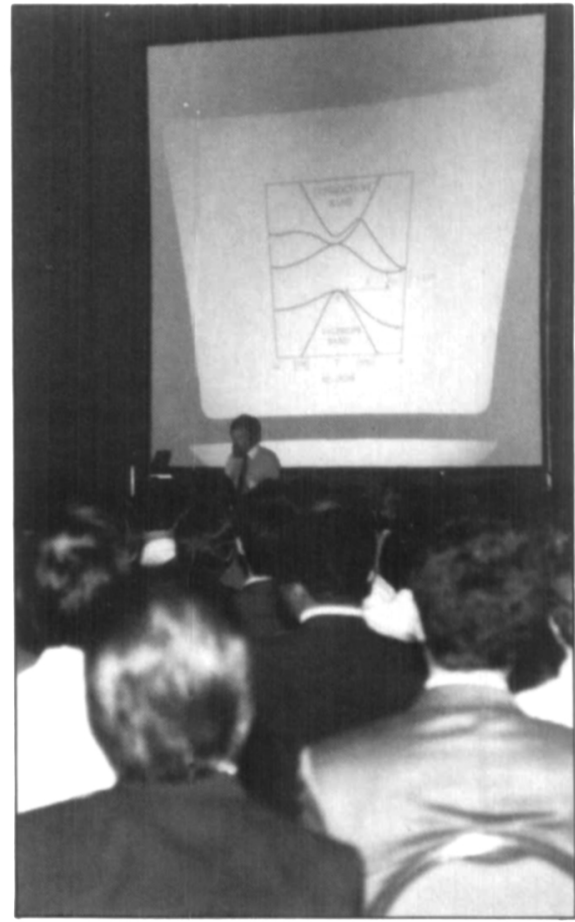

Participants in the special late-news session on visible light from porous silicon hear about the latest progress from researchers at AT\&T Bell Laboratories, RSRE, University of Grenoble, UMIST, and elsewhere. (See sidebar in this report.)

tures with silicon received substantial interest. F. Spaepen (Harvard) cited many examples where the metal layer significantly enhances the silicon crystallization rate. In thin $\mathrm{Al} / \mathrm{Si}$ multilayers, amorphous Si nucleated within the Al layer, leading to full crystallization. K. Tu (IBM T.J. Watson) used the example of whisker growth in $\mathrm{Sn}$ thin films on Si to suggest that in thin films a stress-driven component of atomic diffusion may be as important as the usual components due to chemical potential and concentration gradient.

Much discussion centered on the connection between crystal-to-amorphous solid-state transformations, as observed, for example, when irradiating an alloy with energetic particles or during low-temperature interdiffusion reactions between transition metals, and solid-state melting phenomena. D. Wolf (Argonne National Laboratory, with S. Yip, Massachusetts Institute of Technology) performed computer modeling of melting in elemental crystals. They used an embedded-atom potential for $\mathrm{Cu}$ and the Stillinger-Weber three-body potential for $\mathrm{Si}$. These atomic potentials allow for noncentral forces which must be included in any modeling of melting phe- 
nomena. They find that melting occurs heterogeneously in crystals with defects (at surfaces, voids, or grain boundaries) when the temperature reaches the conventional "thermodynamic melting temperature." In perfect crystals with only point defects, melting is homogeneous and occurs at the higher "mechanical melting temperature" first described by Born. W.L. Johnson addressed crystal-to-amorphous transformations from a more theoretical viewpoint and as it applies to alloys. He described the conditions for polymorphic melting in a binary alloy as a function of temperature, pressure, and alloy composition (work of Pecht, Desre, and Johnson). The model predicts that, as the temperature is decreased, a critical transformation triple point is reached where the crystal (solid solution) coexists with the liquid and amor- phous (glassy) phases of the same composition. At this point the entropy of melting is zero.

Commercial applications of phase transformation are nearing. K. Rubin (IBM Almaden) reviewed optical recording using phase change materials (e.g., $\mathrm{Ge}_{1 \times x \times y} \mathrm{Te}_{\mathrm{x}} \mathrm{Sb}_{\mathrm{y}}$.) Laser-induced transformations between the amorphous phase and the crystalline phase form the basis for this technology. Commercial products have been introduced using this concept but are limited by the thin film's inability to sustain repeated cycling to $\geqslant 10^{6}$ times. The volume change and resulting stress associated with the phase transformation are thought to be the cause of the problem. Nonvolatile random access memories based on ferroelectric films $\left(\mathrm{PbZr}_{1-x} \mathrm{Ti}_{2} \mathrm{O}_{3}\right)$ is generating great interest. The grain size and texture of the perovskite phase critically affect the electrical performance. In vacuum-deposited films, the transformation from the amorphous (as-deposited) state to the pyrochlore phase and then to the perovskite phase is an important materials problem. An alternate preparation technique using sol-gel based coating has been developed, which routinely yields high quality films, as discussed by J. Scott (University of Colorado).

\section{Interplay of Magnetic Properties and Structure Examined}

(See MRS Symposum Proceedings Vol. 232)

The magnetic properties in materials such as permanent magnets, magnetic recording, and magneto-optical recording materials are very sensitive to their microstructures, and so it is vitally important to

\section{Late-News Session Considers Visible Light from Porous Si}

The emission of visible light from silicon under optical and electrical excitation has been the subject of substantial research efforts for decades. A report in the September 3, 1990 issue of Applied Physics Letters, on efficient visible photoluminescence from porous silicon by $L$. Canham's group at the Royal Signals and Radar Establishment (RSRE), United Kingdom, has stimulated a resurgence of interest in this field. The possibility of using silicon structures for optoelectronic applications vastly enhances the potential of directly integrating optoelectronics with VLSI circuitry.

A special symposium at the 1991 MRS Spring Meeting brought together, for the first time, the leaders in this field to report progress and discuss the physics of the emission process. The symposium was organized and chaired by R.F.C. Farrow of IBM's Almaden Research Center and sponsored by Symposium B, Silicon Molecular Beam Epitaxy.

\section{J. Bean (AT\&T Bell Laboratories)} opened the symposium with a review of attempts to achieve light emission from silicon. The indirect band structure of bulk single-crystal silicon prevents effective band-to-band, electron-hole radiative recombination because the transition requires the additional involvement of a phonon (lattice vibration). On the other hand, in direct gap semiconductors such as $\mathrm{GaAs}$ and related materials, recombination can take place directly without phonon involvement. As Bean put it, "It is far harder to get three people together for a meeting than two." Attempts to achieve light emission included doping Si with Er ions and modifying the Si band structure by forming superlattices with $\mathrm{SiGe}$ alloys. None of these approaches was very successful.

Bean showed a scanning electron micrograph of a porous Si surface formed by anodic etching (see photo). The structure clearly showed the long, very narrow isolated crystallites of silicon envisaged in Canham's paper. Bean confirmed that surprisingly bright visible photoluminescence had been observed from porous silicon in the past few weeks at AT\&T Bell Labs. However, the emission was not seen in structures etched to the extreme porosity level $(>90 \%)$ illustrated in the micrograph.

W.Y. Leong of the RSRE team described how the discovery of light emission from porous silicon came out of the attempts to develop silicon-on-insulator (SOI) structures based on oxidation of porous silicon. A combination of anodic and chemical etching was used to generate a highly porous surface layer of silicon. Anodic etching in aqueous $\mathrm{HF}$ solution to $70 \%$ porosity formed an array of pores which were enlarged by subsequent slow chemical etching of the sample in the dark. The remaining silicon material consists of quantumwire type structures which give rise to quantum confinement effects.

By controlling the etching time, the photoluminescence could be shifted from the red to the green wavelength region. This shift is believed to arise from quantum confinement effects due to diminishing dimensions of crystallites in the porous silicon layer. Leong demonstrated the spatial uniformity of emission across the wafer by showing slides of uniform color (green, yellow, orange, red) emission under UV excitation. He pointed out that emission could be obtained both from p-and n-type wafers. Present work at RSRE is aimed at understanding the emission process and achieving electroluminescence.

P. Petroff (University of California, Santa Barbara) suggested a study of the polarization of the photoluminescence to indicate whether the emission was coming from unidirectional quantum wires. He also raised the question of whether the crystal structure of the emitting crystallites was the normal diamond structure or a modified structure.

R. Romestain then described recent developments at the University of Grenoble, France, acknowledging that the work was directly stimulated by the RSRE's published report. Visible emission was immediately found from anodically etched wafers (with varying degrees of porosity) that were already available from prior SOI work! Romestain explained how emission in the 
understand the correlation between them. Symposium T, Magnetic Materials Microstructure and Properties, covered this connection between microstructure and properties and also covered processing and characterization issues which are the key for controlling structure.

The symposium covered magnetic materials with soft, semi-hard, and hard properties, as well as the multilayer thin films in conjunction with their interfacial structures.

One of the recent topics in magnetic materials is the giant magnetic moment material of $\mathrm{Fe}_{16} \mathrm{~N}_{2}$. An invited talk by M. Komuro (Hitachi) summarized the work on $\mathrm{Fe}_{16} \mathrm{~N}_{2}$ films grown by MBE method, which shows $2.9 \mathrm{~T}$ of the saturation magnetization, much higher than for $\mathrm{FeCo}$, as reported by Kim and Takahashi in 1972. T.
Yogi (IBM) described the high density recording materials of $\mathrm{CoPtCr}$ films in connection with columnar structures. He showed how the columnar structures are influenced by the sputtering conditions, which in turn relates directly to recording performance.

M. Takahashi (Tohoku University) described the soft magnetic properties in Sendust and Fe-N films. A "very" soft magnetic property through nanocrystalline state was discussed based on a so-called ripple theory. It is of interest that the permeability and coercive force are shown to be closely related to a grain size. K. Mohri (Nagoya University) described the various applications of magnetic materials as sensors, including a sensor that detects human sleepiness.

A new characterization technique for studying magnetic materials is spin polarized low energy electron microscopy (SPLEEM). This technique can detect the magnetic domain very near the surface of a magnetic material with a resolution better than $500 \AA$. M. Altman (Technische Universität Clausthal) presented a new result of Co single crystals, a clear picture of the domain pattern in the hexagonal plane of Co. I.R. McFadyen (IBM) discussed the use of electron microscopy, in particular differential phase contrast, for the domain observation. He showed the change in domain pattern in a $\mathrm{Co}$ hexagonal plane as a function of temperature, demonstrating the powerful advantage of differential phase contrast. visible could be obtained by etching processes somewhat different to those described by Leong. After anodic etching to a porosity of $85 \%$, the chemical etch speed could be greatly increased by illumination. The result was similar to that reported by the RSRE group. An alternative scheme anodically etched wafers to $65 \%$ porosity in ethanoic $\mathrm{HF}$ solution followed by anodic oxidation in $0.1 \mathrm{M} \mathrm{KNO}_{3}$ solution. This process generated oxide-covered sidewalls on the silicon wires and could progressively convert more of the silicon to oxide, leading to good control over the emission wavelength and much more stable visible emission intensity than etching alone.

Interesting data were reported on emission decay after pulsed excitation with a Q-switched Nd:YAG laser. A very rapid (ns) rise time followed by a much slower decay $(\sim \mu \mathrm{s})$ was found, with the decay times varying from $100 \mu$ s at 900 $\mathrm{nm}$ to $5 \mu \mathrm{s}$ at $550 \mathrm{~nm}$ emission wavelength. These lifetimes are very long compared with those for a direct gap recombination process, suggesting that another process is determining the lifetime, perhaps electron tunneling through a potential barrier to a quenching site. Romestain envisaged a spatially varying band gap in the silicon quantum wires which vary in dimensions along their length. These structures might be more accurately described as interconnected quantum dots of varying size.

Romestain also revealed the observa-

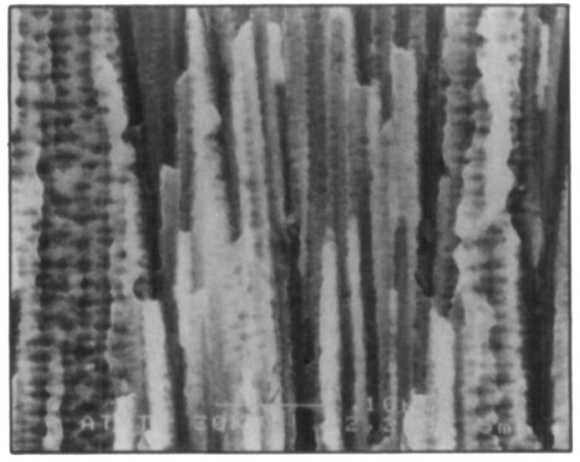

Cross-sectional view of porous $n$-type silicon taken with a JEOL 6300 scanning electron microscope with a field emission source. Note: This is a nonluminescent sample. Photo courtesy of J. Macaulay and J. Bean, ATET Bell Laboratories.

tion of electroluminescence from the porous silicon. During anodic etching, visible light emission was detected until the etching self-terminated. Leong confirmed that electroluminescence had also been achieved at RSRE but would not elaborate further.

In the final talk, A. Pinczuk (AT\&T Bell Laboratories) reviewed methods of engineering regular arrays of quantum wires in GaAs and related coompounds.

Audience discussion was led by $W$. Lenth of IBM Almaden Research Center. T. Peaker (UMIST, Manchester, U.K.) reported studies of the emission's temperature dependence. On cooling, the emission intensity was found to increase by two orders of magnitude. This suggests an upper limit of $1 \%$ for photoluminescence efficiency at room temperature.

Lenth concluded the session by summarizing areas to be clarified before applications can be considered, including quantitative measurements of the efficiency of the emission process and further time-resolved studies of the recombination process. The evidence for a quantum size effect was compelling but indirect. Direct imaging of the quantum wires by microscopy would be informative.

The overall impression from the session was that of a spectacular phenomenon with interesting application potential, but requiring many more controlled experiments to understand the underlying physics.

The simplicity of wafer processing makes these studies accessible to many groups. The fact that several independent groups have rapidly reproduced visible emission from porous silicon supports this view. However, as Pinczuk pointed out, it would be easier to understand the physics of the emission if the size and distribution of the silicon quantum wires could be controlled, as with the compound semiconductors. Clearly, this will be one of many lines of attack in this exciting new field.

R.F.C. Farrow 